\title{
La resolución de problemas como forma de aprendizaje del Derecho Civil
}

\section{Problem solving as a way of learning Civil Law}

Manuel García Mayo

ORCID: https://orcid.org/0000-0003-4647-0158

Universidad de Sevilla

Departamento de Derecho Civil

e Internacional Privado

mgarcia127@us.es

DOI: http://dx.doi.org/10.12795/9788447231003.023

Pp.: 490-514 


\section{Breve descripción del contexto}

El presente ciclo de mejora (en adelante, CIMA) ha sido diseñado para la asignatura de Derecho Civil III (Derechos reales e hipotecario), que se imparte en el tercer curso del Grado en Derecho de la Universidad de Sevilla. El CIMA se aplica a lo largo de dos semanas - un total de ocho horasen un grupo que, aun siendo numeroso -más de setenta alumnos- son pocos los que acuden a clase en tanto que está conformado por muchos alumnos repetidores.

Las clases se desarrollan en la propia Facultad de Derecho, en horario de tarde, situándose el profesor sobre una tarima en la que se encuentra la mesa con el ordenador, una pizarra y una pantalla en la que poder proyectar imágenes del ordenador o de cualquier otro dispositivo electrónico, y los alumnos sentados en largas bancadas fijadas al suelo, sin posibilidad, por tanto, de ser movidas a fin de cambiar, en un caso hipotético, la distribución del aula.

No obstante, la circunstancia más relevante - por su novedad - a tener en cuenta en el desarrollo de este CIMA -al igual que en el de todos los demás aplicados en el curso 2020/2021- es la situación excepcional en la que nos encontramos a consecuencia de la pandemia de COVID-19. La emergencia sanitaria existente a nivel mundial en general, y en nuestro país en particular, ha conllevado la adopción de importantes medidas de seguridad a fin de evitar el contagio entre los ciudadanos. Esas medidas han tenido, obviamente, un importante reflejo en un colectivo tan amplio y con tanta actividad como es el universitario. Así, tras una primera semana de docencia completamente online, y a fin de garantizar la preceptiva distancia de seguridad entre alumnos, cada grupo de la Facultad de Derecho se dividió en tres subgrupos ( $a, b$ y c), aplicándose un sistema bimodal de docencia con una asistencia presencial rotatoria por parte de los alumnos: cada semana del curso, uno de los subgrupos tenía la oportunidad de asistir presencialmente a clase - según el orden preestablecido-, mientras que los otros dos subgrupos podían seguir las clases en streaming desde sus casas.

Ciclos de Mejora en el Aula (2020). Experiencias de Innovación Docente de la US Esta obra se distribuye con la licencia Creative Commons 
Quienes seguían la clase desde sus casas lo hacían a través de la plataforma Blackboard Collaborate, plataforma que la Universidad de Sevilla pone a disposición de toda su comunidad y que ofrece la posibilidad de interactuar con los alumnos a través de audio, video, archivos compartidos, pizarra electrónica, etc.

En el aula también se presentaban muchas novedades: necesidad de que el profesor limpiase toda la zona de trabajo suya con carácter previo al comienzo de la clase, uso obligatorio por alumnos y profesores tanto de geles hidroalcohólicos como de mascarillas, necesidad de impartir la docencia con puertas y ventanas abiertas para facilitar la ventilación... Todo ello con el objetivo principal de evitar cualquier tipo de contagio entre los asistentes.

Todo este cúmulo de circunstancias llevaron a que el desarrollo de las clases en general y de este CIMA en particular fuesen especialmente complejos. En primer lugar, la preparación de todo el sistema informático para desarrollar la clase bimodal, así como la limpieza de la zona de trabajo del profesor, suponía una importante pérdida de tiempo, muy notable en aquellas clases cuya duración era únicamente de una hora. En segundo lugar, la interacción entre profesor y alumnos resultaba especialmente compleja: los alumnos presentes en el aula debían estar separados, al menos, dos metros, sin posibilidad de que pudieran reunirse ni tan siquiera para trabajar en alguna actividad concreta; el uso de mascarillas dificultaba tanto la expresión verbal como la no verbal que se produce en cualquier clase usual; los alumnos que seguían la clase desde casa, motivado por la no presencialidad, eran menos propensos a intervenir en la clase, a lo que se une que todo cuanto hablasen los compañeros presentes en el aula no era perceptible por los alumnos que estaban en casa.

Ciclos de Mejora en el Aula (2020). Experiencias de Innovación Docente de la US Esta obra se distribuye con la licencia Creative Commons 
Todas las características descritas han influido, irremediablemente, tanto en el diseño como en la aplicación del CIMA que aquí se presenta. Muy probablemente, si este hubiera sido un curso corriente, aun partiendo de la misma base - de la misa idea de cambio metodológico-, tanto el diseño del CIMA como su puesta en práctica habrían sido sustancialmente diferentes.

\section{Diseño previo del CIMA}

\section{Mapa de contenidos}

Atendiendo a las circunstancias referidas anteriormente, así como al espacio temporal en el que desarrollaré el CIMA, diseño este último partiendo de dos temas concretos muy importantes en el programa de la asignatura: el referente a la posesión y el referente al derecho real de propiedad. Una vez identificado ambos temas como materia objeto de estudio en estas semanas, intento realizar un mapa de contenido en el que se vea claramente la interrelación entre ambos conceptos y, con ello, de toda la materia que se estudia con ocasión de los mismos. Utilizo tanto el diseño en sí, como los colores y las flechas, para que se vean plasmadas en la pantalla esa interrelación que, sin contar con este recurso, sería complicado de apreciar en abstracto.

La materia aludida, correspondientes a estas dos semanas, la divido a su vez en cinco bloques. Los dos primeros bloques darán una respuesta concisa a una de las grandes preguntas: ¿qué es la posesión? Los bloques tercero y cuarto otorgarán, por su parte, respuesta, a la segunda gran pregunta: ¿qué es la propiedad? Cada uno de estos cuatro bloques, da respuesta, asimismo, a una

Ciclos de Mejora en el Aula (2020). Experiencias de Innovación Docente de la US Esta obra se distribuye con la licencia Creative Commons 
primera pregunta inicial de tipo eminentemente práctico, que le planteo a los alumnos al comienzo de cada uno de los bloques. El estudio de los cuatro bloques referidos servirán para poder estudiar y dar respuesta a dos importantes materias: la adquisición a non domino y la usucapión; el estudio de ambas figuras se comienzo, asimismo, con la formulación de dos preguntas de tipo eminentemente práctico, como puede observarse en el mapa de contenido (vid. figura 1).

Hasta la aplicación del presente CIMA, la docencia, por mi parte, atendía, fundamentalmente, al sistema clásico de clase magistral, independientemente de alguna clase que dedicase a la realización y resolución de ejercicios prácticos con carácter esporádico. Es por ello que el objetivo esencial de este ciclo de mejora ha sido el de, sin abandonar radicalmente la clase magistral -que en esta disciplina la considero esencial-, restar peso a la misma en un nuevo diseño de las clases en las que perseguía un doble objetivo: en primer lugar, convertir al alumno en protagonista de la misma; en segundo lugar, basar la clase en el planteamiento y resolución de problemas que resulten de interés para el alumno.

Ciclos de Mejora en el Aula (2020). Experiencias de Innovación Docente de la US Esta obra se distribuye con la licencia Creative Commons 


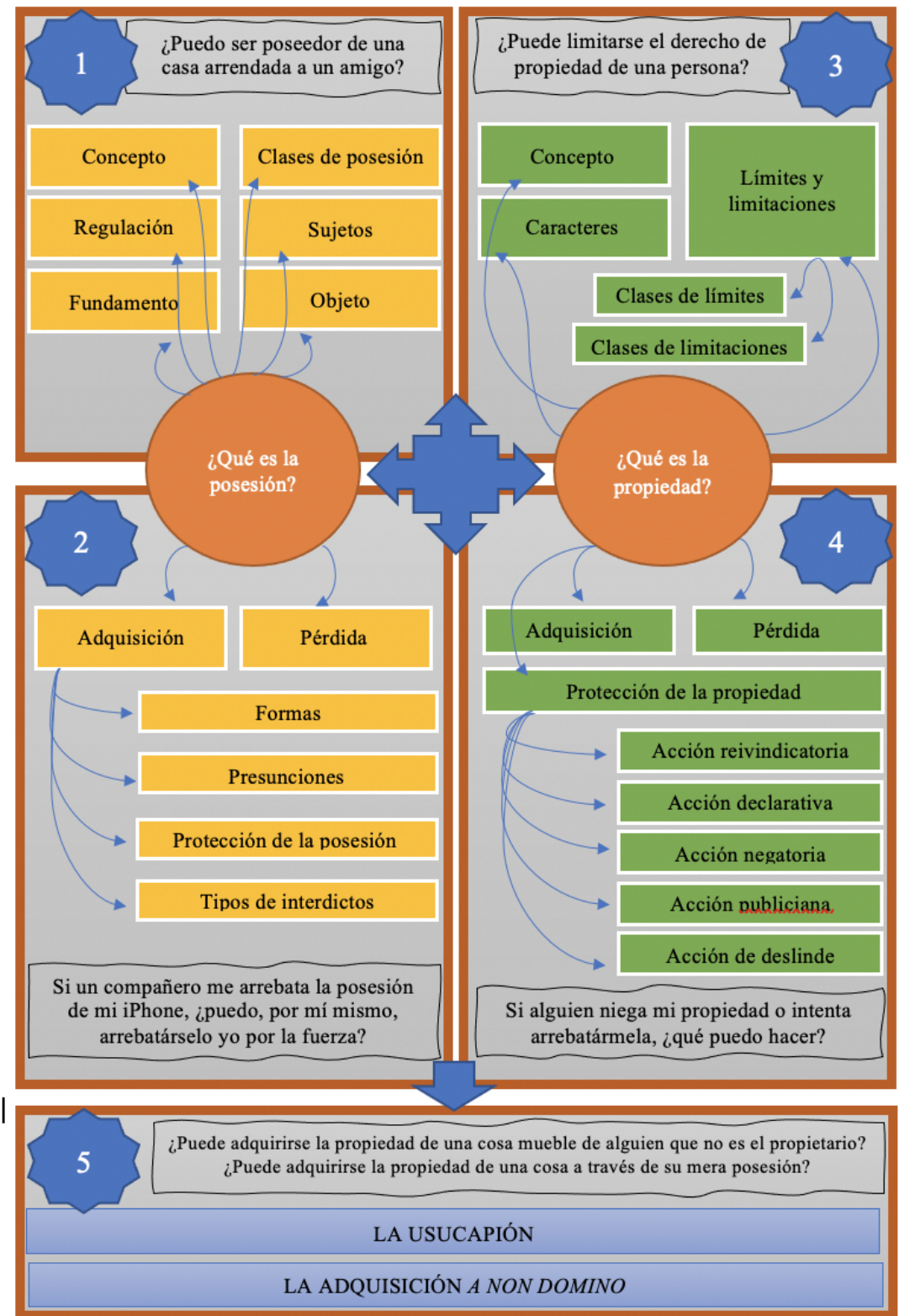

Figura 1. Mapa de contenidos

Ciclos de Mejora en el Aula (2020). Experiencias de Innovación Docente de la US Esta obra se distribuye con la licencia Creative Commons Reconocimiento-NoComercial-SinObraDerivada Internacional (CC BY-NC-ND 4.0.) 
Teniendo en cuenta el mapa de contenidos planteado y el objetivo perseguido con la aplicación de este CIMA, cada uno de los cinco bloques en los que se divide el contenido de la materia a impartir en estas dos semanas se abre con una pregunta práctica que invite a los alumnos a reflexionar y a percatarse de que, sin el conocimiento necesario, no son capaces de dar respuesta al problema o, al menos, de hacerlo de forma acertada. Se trata, en definitiva, no solo de que observen la utilidad práctica de las clases, sino de captar su atención desde un inicio a fin de poder resolver por ellos mismos, al final de la explicación, ese problema planteado y que, sin el conocimiento necesario, no habrían sido capaces de resolver.

Tabla 1. Secuencia de actividades programadas

\begin{tabular}{|c|l|l|}
\hline Núm. de sesión & Núm. de actividad & \multicolumn{1}{|c|}{ Descripción de la actividad } \\
\hline \multirow{2}{*}{$\begin{array}{c}\text { SESIÓN 1 } \\
\text { (1 hora) }\end{array}$} & Actividad 1 & $\begin{array}{l}\text { Realización, por parte de los } \\
\text { alumnos, del cuestionario inicial en } \\
\text { horario de clase. }\end{array}$ \\
\cline { 2 - 4 } & Actividad 2 & $\begin{array}{l}\text { Proposición a los alumnos de una } \\
\text { actividad a desarrollar a través de la } \\
\text { red social Twitter. }\end{array}$ \\
\hline \multirow{2}{*}{$\begin{array}{c}\text { SESIÓN 2 } \\
\text { (1 hora) }\end{array}$} & Actividad 3 & $\begin{array}{l}\text { Explicación teórica del cuadro } \\
\text { número 1 del mapa de contenidos, } \\
\text { a partir de un problema práctico } \\
\text { planteado. }\end{array}$ \\
\hline \multirow{3}{*}{$\begin{array}{l}\text { SESIón 3 } \\
\text { (2 horas) }\end{array}$} & Actividad 4 & $\begin{array}{l}\text { Explicación teórica del cuadro } \\
\text { número 2 del mapa de contenido, } \\
\text { a partir de un problema práctico } \\
\text { planteado. }\end{array}$ \\
\cline { 2 - 4 } & Actividad 5 & $\begin{array}{l}\text { Explicación teórica del cuadro } \\
\text { número 3 del mapa de contenido, } \\
\text { a partir de un problema práctico } \\
\text { planteado. }\end{array}$ \\
\hline
\end{tabular}

Ciclos de Mejora en el Aula (2020). Experiencias de Innovación Docente de la US Esta obra se distribuye con la licencia Creative Commons 


\begin{tabular}{|c|c|c|}
\hline $\begin{array}{c}\text { SESIÓN } 4 \\
\text { (1 hora) }\end{array}$ & Actividad 6 & $\begin{array}{l}\text { Explicación teórica del cuadro } \\
\text { número } 4 \text { del mapa de contenido, } \\
\text { a partir de un problema práctico } \\
\text { planteado. }\end{array}$ \\
\hline \multirow[b]{2}{*}{$\begin{array}{c}\text { SESIÓN } 5 \\
\text { (1 hora) }\end{array}$} & Actividad 7 & $\begin{array}{l}\text { Realización por los alumnos de un } \\
\text { caso práctico proporcionado por } \\
\text { el profesor para su resolución en } \\
\text { base a la teoría explicada hasta el } \\
\text { momento. }\end{array}$ \\
\hline & Actividad 8 & $\begin{array}{l}\text { Reflexión y trabajo común a fin de } \\
\text { intentar dar repuesta a las preguntas } \\
\text { planteadas en el cuadro número } \\
5 \text {, con lo que se irá respondiendo, } \\
\text { a su vez, al supuesto práctico } \\
\text { proporcionado a los alumnos en } \\
\text { esta misma sesión. }\end{array}$ \\
\hline \multirow{4}{*}{$\begin{array}{l}\text { SESIÓN } 6 \\
\text { (2 horas) }\end{array}$} & Actividad 9 & $\begin{array}{l}\text { Continuación y conclusión de la } \\
\text { explicación que da respuesta a las } \\
\text { preguntas del cuadro } 5 \text {. }\end{array}$ \\
\hline & Actividad 10 & $\begin{array}{l}\text { Apertura de un tiempo de debate } \\
\text { y comentarios sobre la actividad } \\
\text { desarrollada a través de la } \\
\text { plataforma Twitter durante estas } \\
\text { dos semanas. }\end{array}$ \\
\hline & Actividad 11 & $\begin{array}{l}\text { Realización del cuestionario final, a } \\
\text { fin de comparar resultados respecto } \\
\text { al cuestionario inicial. }\end{array}$ \\
\hline & Actividad 12 & $\begin{array}{l}\text { Ejercicio tipo test cuantificable en la } \\
\text { nota final de la asignatura. }\end{array}$ \\
\hline
\end{tabular}

\section{Cuestionario inicial-final}

Las que se exponen a continuación son las preguntas planteadas a los alumnos tanto en el cuestionario inicial al comienzo del CIMA, como en el cuestionario final a la conclusión del mismo. Como puede observarse, las preguntas que se formulan coinciden, en gran medida, con las que han servido de sustento para configurar y trazar el mapa de contenido diseñado. Los propios alumnos, con el desarrollo de las explicaciones a lo largo de estas dos semanas, irán comprobando por sí mismos hasta qué punto

Ciclos de Mejora en el Aula (2020). Experiencias de Innovación Docente de la US Esta obra se distribuye con la licencia Creative Commons 
tenían razón o no en cuantas cuestiones respondieron en el cuestionario inicial.

Entre estas preguntas, las hay de contenido muy práctico en concreto y también hay otras que están planteadas desde un tono más reflexivo, a fin de que el alumno piense y medite una respuesta que puede tener diferentes interpretaciones y que está sometida a debate. Son, en gran parte, preguntas en las que la lógica de una persona que desconoce la teoría de la asignatura le llevaría a responder de forma totalmente opuesta a como lo haría tras un conocimiento exhaustivo de la materia. Es decir, la lógica por la que se guiarán en el cuestionario inicial les llevará a respuestas bastante distantes de las que corresponden aplicando los fundamentos jurídicos a estudiar.

Tabla 2. Cuestionario inicial - final

\begin{tabular}{|c|l|}
\hline Pregunta 1 & ¿Qué es la posesión? \\
\hline Pregunta 2 & ¿Qué es la propiedad? \\
\hline Pregunta 3 & $\begin{array}{l}\text { ¿Puedo tener yo la posesión de una casa que tengo } \\
\text { arrendada a un amigo mío? }\end{array}$ \\
\hline Pregunta 4 & $\begin{array}{l}\text { Si un compañero me arrebata la posesión de mi iPhone, } \\
\text { ¿puedo, por mí mismo, arrebatárselo yo por la fuerza? }\end{array}$ \\
\hline Pregunta 5 & $\begin{array}{l}\text { ¿Puede limitarse el derecho de propiedad de una } \\
\text { persona? En su caso, ¿Cuándo estaría justificado? }\end{array}$ \\
\hline Pregunta 6 & $\begin{array}{l}\text { La atribución del uso de la vivienda familiar al cónyuge } \\
\text { que no es propietario una vez sobrevenido el divorcio, } \\
\text { ¿considera que es una limitación de la propiedad? ¿Por } \\
\text { qué? }\end{array}$ \\
\hline Pregunta 7 & $\begin{array}{l}\text { Limitar el precio del alquiler, ¿considera que es una } \\
\text { limitación del derecho de propiedad? ¿Por qué? }\end{array}$ \\
\hline Pregunta 8 & $\begin{array}{l}\text { Si alguien niega mi propiedad o intenta arrebatármela, } \\
\text { ¿qué puedo hacer? }\end{array}$ \\
\hline Pregunta 9 & $\begin{array}{l}\text { ¿Puede adquirirse la propiedad de una cosa mueble de } \\
\text { alguien que no es el propietario? ¿Cuál cree que es el } \\
\text { fundamento de que eso sea así? }\end{array}$ \\
\hline Pregunta 10 & $\begin{array}{l}\text { ¿Puede adquirirse la propiedad de una cosa a través de } \\
\text { su mera posesión? ¿Cuál cree que es el fundamento de } \\
\text { que eso sea así? }\end{array}$ \\
\hline
\end{tabular}

Ciclos de Mejora en el Aula (2020). Experiencias de Innovación Docente de la US Esta obra se distribuye con la licencia Creative Commons 


\section{Aplicación del CIMA}

\section{Descripción del desarrollo de las sesiones}

\section{Sesión 1 (1 hora)}

\section{Actividad 1: cuestionario inicial}

La primera sesión de las seis en las que voy a aplicar el CIMA, y que suman un total de ocho horas, la comienzo explicando brevemente a los alumnos el motivo del cambio que van a experimentar en la dinámica habitual de las clases. En este sentido, les hago saber el curso de formación del profesorado en el que me encuentro inmerso y cómo pretendo aplicar un ciclo de mejora en nuestras clases a fin de experimentar un cambio innovador a la hora de impartir la docencia, con el fin principal de que ellos sean más partícipes y de que, en definitiva, acaben aprendiendo de una forma diferente, resultado de la cual, más que memorizar, interiorizarán ideas y conceptos y sabrán relacionarlos para poder dar respuesta, así, a los interrogantes que se vayan planteando a lo largo de estas dos semanas de clases.

Tras la referida y breve explicación, procedo a realizar el cuestionario inicial. Hemos de partir de que nos encontramos ante una docencia bimodal, en la que parte de los alumnos se encuentran en casa, siguiendo la case streaming, y otra parte se encuentra presencialmente en el aula, dándose la circunstancia que solo son cinco o seis alumnos máximos los que acuden presencialmente al aula. Atendiendo a tales circunstancias, decido que todos los alumnos realicen el cuestionario a través de la herramienta "Actividad" de la plataforma virtual. De esta forma, todos lo harán en igualdad de condiciones y evito el traspaso de papeles entre profesor y alumnos, lo cual 
se encuentra especialmente desaconsejado a fin de vitar la propagación de la pandemia. Les concedo cuarenta minutos para que realicen el test, haciendo especial hincapié en que reflexionen y argumenten el porqué de sus respuestas.

La sensación, por parte de los alumnos asistentes fue de sorpresa, y quizás de temor, ante el cambio de la dinámica imperante hasta el momento que les posibilitaba la toma de apuntes. No obstante, los animé a que confiaran en este nuevo diseño de clase para, posteriormente, valorar entre todos si era más óptimo este sistema o el seguido hasta entonces.

Actividad 2: Twitter como herramienta para la docencia

En la última parte de la clase, propongo a los alumnos una nueva actividad, a desarrollar a través de la red social Twitter. El profesor, en primer lugar, indica su perfil en esta red social (@manuel_mayo_), al cual los alumnos que deseen realizar esta actividad deberán seguir. Les explico que la idea es que el profesor vaya publicando, a lo largo de las dos semanas: píldoras informativas sobre la materia objeto de explicación; sentencias interesantes al respecto; noticias de la prensa que estén relacionadas; e incluso lanzará preguntas a las que los alumnos podrán responder e interactuar entre ellos. A fin de motivarles con la posibilidad de obtener una rendimiento cuantitativo que se refleje a efectos de calificación, les informo, asimismo, de que toda la información compartida a través de esta herramienta dará pistas sobre algunas de las preguntas que se plantearán en algunas de las actividades programadas de evaluación continua. Todos los Tweets se publicarán bajo el hashtag \#Civil3US. La participación en Twitter bajo esta etiqueta se computará, a todos los efectos, como participación en clase.

La iniciativa es asumida con bastante agrado por parte del alumnado, hasta el punto de que, antes de concluir la clase, muchos de ellos ya me seguían en la red social. Lo

Ciclos de Mejora en el Aula (2020). Experiencias de Innovación Docente de la US Esta obra se distribuye con la licencia Creative Commons 
consideraron, pues, como una muestra de acercamiento hacia ellos, en tanto que íbamos a trabajar con una herramienta a la que ellos están sobradamente habituados fuera del aula, pero que aún no habían usado como herramienta de trabajo.

\section{Sesión 2 (1 hora)}

Actividad 3: explicación teórica del cuadro número 1

Esta segunda sesión la comienzo proyectándole a los alumnos el mapa de contenido realizado para este CIMA y explicando la interrelación que existe entre todos los conceptos que lo componen y cómo el conocimiento de todos ellos nos posibilitarán dar respuesta a las dos preguntas con las que se encabeza el bloque 5.

Se abre, así, la clase con el planteamiento de la primera pregunta de tipo práctico a la que se dará respuesta con la explicación de esta sesión: ¿Puedo tener yo la posesión de una casa que tengo arrendada a un amigo mío? Una pregunta que, a priori, y antes de conocer la materia, cualquiera respondería negativamente, porque va contra el sentido de la lógica. Empiezo pues, no solo planteando la pregunta, sino instando a los alumnos a que intenten contestarla. Efectivamente, la totalidad de las respuestas fueron en sentido negativo, y fue ello lo que me dio lugar a empezar la explicación de esta primera parte del mapa de contenido que, precisamente por ser la inicial, es, quizás, la más árida o puramente teórica. Tras el desarrollo de la explicación, ilustrada con ejemplos prácticos, que resultan cotidianos para ellos, vuelvo a plantear la pregunta y el resultado, evidentemente, es radicalmente diferente al del inicio de la clase.

\section{Sesión 3 ( 2 horas)}

Actividad 4: explicación teórica del cuadro número 2

Comienzo la clase con el planteamiento de la segunda de las preguntas planteadas en el mapa de contenido, 
correspondiente con el segundo cuadro del referido mapa, y a la que se pretende dar respuesta con la explicación de esta primera parte de la clase: Si un compañero me arrebata la posesión de mi iPhone, ¿puedo, por mí mismo, arrebatárselo yo por la fuerza? Planteada la pregunta y pidiéndole a los alumnos su primera impresión al respecto, ocurre algo similar a cuanto acontecía con la primera de las preguntas planteadas en la sesión anterior: la lógica les induce a pensar que, obviamente sí, aunque en esta sesión ya son más conscientes de que, probablemente la lógica no les conduzca a buen puerto.

Suscitado un breve debate sobre quienes pensaban de una forma o de otra, y con el interés suscitado entre ellos por conocer la respuesta definitiva, comienzo la explicación de la segunda parte de las cinco en las que se divide la explicación teórica de estas dos semanas, la cual acompaño en todo momento con sentencias que -por cercanía-considero podrían ser de su interés, así como con noticias de prensa de los últimos meses que pudieran estar relacionadas con la materia. Con la explicación de estas dos primeras partes del mapa de contenido, se da respuesta, a su vez, al primer gran interrogante teórico planteado en el referido mapa: ¿Qué es la posesión? Se consigue, así, dar respuesta a este gran interrogante puramente teórico que, pese a su complejidad y carácter teórico, supone uno de los puntos más importantes del temario con importantes consecuencias prácticas.

Actividad 5: explicación teórica del cuadro número 3

Tras un breve descanso en mitad de la clase, teniendo en consideración que estamos ante una sesión de dos horas, procedo a plantear la tercera de las preguntas prácticas del mapa de contenido a la que intentaremos dar respuesta con la explicación de la tercera parte del mapa de contenido: ¿Puede limitarse el derecho de propiedad de una persona?

Ciclos de Mejora en el Aula (2020). Experiencias de Innovación Docente de la US Esta obra se distribuye con la licencia Creative Commons 
Es una pregunta especialmente abierta, sobre todo antes de conocer el contenido teórico que se va a explicar. Tan es así que la pregunta da origen a un interesante debate entre aquellos que consideran que el poder del propietario es ilimitado y aquellos otros que consideran, contrariamente, que existen limitaciones al derecho de propiedad y se aventuran, incluso, a ofrecer algunos ejemplos.

Con el debate abierto, procedo, pues, a explicar la tercera parte de la materia programada para estas dos semanas. Esta explicación teórica, siempre apoyada en supuestos prácticos y en sentencias con supuestos de hecho que puedan suscitar interés al alumnado, no solo dará respuesta a la pregunta planteada sobre los límites al derecho de propiedad que, como les explico, se van adaptando al paso del tiempo, sino que, junto con la próxima sesión, dará respuesta a la otra gran pregunta planteada en el mapa de contenido: ¿qué es la propiedad?

\section{Sesión 4 (1 hora)}

Actividad 5: explicación teórica del cuadro número 4

Comienzo la sesión con el planteamiento de la cuarta de las preguntas prácticas a la que se dará respuesta a través de la explicación correspondiente a esta sesión: Si alguien niega mi propiedad o intenta arrebatármela, ¿qué puedo hacer yo?

Es, quizás, la pregunta más complicada de cuantas se han planteado hasta ahora, por ser la más técnica, pero, aún así, por el hecho de encontrarnos ya en esta dinámica desde hace varias clases, los alumnos se animan a responder y se aventura a decir distintas acciones que, judicialmente, consideran que se podrían emprender a efectos de proteger el derecho de propiedad. No obstante, no dejan de surgir dudas relacionadas con la forma en la que alguien puede negar mi propiedad o puede arrebatármela. Es más, no alcanzar a imaginarse que eso pueda ocurrir en la realidad.

Ciclos de Mejora en el Aula (2020). Experiencias de Innovación Docente de la US Esta obra se distribuye con la licencia Creative Commons 
Una vez, pues, que les ha suscitado interés práctico la materia, procedo a explicar el contenido relativo a esta sesión. Comienzo haciendo referencia a las formas a través de las cuales un sujeto puede adquirir la propiedad. Una vez sabemos cómo se puede llegar a ser propietario, vemos, entonces, de qué forma puedo proteger ese derecho de propiedad del que soy titular, de forma que vemos las distintas acciones que se pueden emprender y las diferencias entre ellas, ejemplificándolas siempre con supuestos prácticos: acción reivindicatoria, acción declarativa, acción negatoria, acción publiciana y acción de deslinde. Finalmente, explico de qué forma podría llegar a perderse la propiedad.

\section{Sesión 5 (1 hora)}

Actividad 6: repaso de la teoría explicada

Al comienzo de la clase, y teniendo en cuenta que ya se ha explicado gran parte de la teoría, concretamente la que corresponde a los cuatro primeros cuadros o apartados en que se divide el mapa de contenidos, realizo un breve resumen a modo de conclusión de lo estudiado en las tres últimas sesiones, apoyándome, en todo momento, en el mapa de contenido que tienen proyectado en la clase y en la plataforma virtual, a efectos de que, más allá del a explicación independiente de cada una de las sesiones, observen la interrelación entre todo lo explicado.

\section{Actividad 7: realización de caso práctico}

Con toda la teoría explicada en las sesiones anteriores, que al comienzo de la clase hemos repasado brevemente, y detectadas las interrelaciones entre todo lo abordado, la idea es dar respuesta, finalmente, a las dos grandes preguntas con las que se cierra el mapa de contenido (cuadro 5 del mapa de contenido) y que están referidas a las dos cuestiones más importantes del temario proyectado para estas dos semanas: la adquisición a non domino y la usucapión.

Ciclos de Mejora en el Aula (2020). Experiencias de Innovación Docente de la US Esta obra se distribuye con la licencia Creative Commons 
No obstante, y antes de abordar esas dos preguntas y la explicación de las cuestiones correspondientes, procedo a realizar una especie de clase invertida, en el sentido de que en lugar de explicar la teoría y hacer posteriormente un caso práctico, comienzo encargándoles, en primer lugar, un caso práctico a realizar en clase.

La idea es, además, que sea un caso práctico de tremenda actualidad, a fin de suscitar especialmente su interés. Para ello les proporciono, a través de la plataforma, un caso práctico real y que ha sido muy mediático en las últimas semanas: la recuperación por el Estado del Pazo de Meirás, que venía poseyendo la familia de Francisco Franco. Concretamente de las dos grandes cuestiones que nos quedan pendientes, la que se aborda en este caso práctico es la usucapión.

Al hilo del supuesto que les proporciono, les planteo distintas preguntas: ¿es válida la donación que se realizó del Pazo de Meirás a Francisco Franco? ¿Es válida la compraventa que se realiza posteriormente también a Francisco Franco? ¿Por qué dice el Estado, entonces, que le pertenece la propiedad del edificio? ¿Podría utilizar ese mismo argumento la familia Franco? ¿Por qué?

A efectos de conseguir que se relacionen los alumnos y que discutan entre ellos y lleguen a conclusiones en común, organizo a los 16 alumnos presentes en cuatro grupos de cuatro alumnos, cada uno de los cuales trabaja en una sesión independiente de Blackboard. Yo, como profesor, me voy pasando por cada una de ellas para escuchar cuanto dicen y plantearles, al hilo de lo que escucho, otras posibles preguntas. Los cuatro alumnos que tengo presentes en clase, forman un grupo presencial entre ellos con las medidas y cautelas pertinentes atendiendo a la situación sanitaria.

Los últimos quince minutos de la clase los dedico a que cada grupo, a través de un portavoz, exponga las conclusiones a las que han llegado en alguna de las preguntas que he formulado. Lo cierto es que, pese a no haberse

Ciclos de Mejora en el Aula (2020). Experiencias de Innovación Docente de la US Esta obra se distribuye con la licencia Creative Commons 
explicado aún la materia propia de las dos grandes cuestiones que se incluyen en el apartado cinco del mapa de contenido, en cierta medida, se acercan a dar la respuesta correcta, y lo hacen sirviéndose de lo que ya han viso en clases anteriores y que, según lo antedicho, es lo que sustenta el resto de la explicación que queda pendiente. No obstante, existen grandes discrepancias entre los grupos y muestran un especial interés por recibir la explicación teórica y saber, así, cuál de ellos ha acertado en sus respuestas.

\section{Sesión 6 ( 2 horas)}

Actividad 8: explicación teórica del cuadro número 5

Comienzo esta sesión de dos horas con la explicación de las dos cuestiones que quedan pendientes de la teoría programada: la adquisición a non domino y la usucapión. En primer lugar, explico la usucapión, al hilo del supuesto práctico que planteamos en la última sesión. Con la explicación, y las respuestas que ellos dieron en la clase anterior y que fueron oportunamente anotadas por mí, acudimos a la sentencia del Juzgado de Primera Instancia que ha resuelto sobre el conflicto existente en torno al Pazo de Meirás, y comprobamos, efectivamente, si lo que los alumnos respondieron en la clase anterior estaba en sintonía con lo resuelto por el juzgado. Intentamos, asimismo, identificar, qué argumentos podría seguir manteniendo la familia Franco a fin de recurrir la sentencia y acudir a segunda instancia.

Posteriormente procedo a la explicación de la adquisición a non domino. Lo hago de una forma peculiar: comienzo poniendo en los altavoces del aula (escuchándose también en casa) la marcha "Amarguras" del compositor Font de Anta, sin compartir la pantalla de mi ordenador, por lo que no sabían que era yo quien estaba poniendo la música. Los alumnos, en clase, se empezaban a mirar entre ellos y el que identificaba la marcha reía tímidamente. 
¿Quién ha puesto "Amarguras"? -escribió alguien por el chat de la plataforma. "Yo" - contesté-. A lo que siguió una reacción de sorpresa por gran parte de los alumnos. Con la marcha "Amarguras" sonando de fondo, expliqué esta figura. La razón de ello era que la explicación la fundamenté en un ejemplo relacionado con la Semana Santa de Sevilla y que tenía como protagonista el cartel de la Semana Santa, que fue vendido a un tercero por el dueño del comercio donde se exponía, sin autorización de su autora.

Actividad 9: comentarios de la actividad desarrollada en Twitter

En esta segunda parte de la clase procedemos a realizar algunos comentarios respecto a lo que han compartido tanto el profesor como distintos alumnos a través de esta red social bajo la etiqueta prefijada \#Civil3US. Comentamos, pues, en clase, algunas sentencias y noticias de prensa que se compartieron durante esos días, así como comentarios de distintos juristas que fueron retuiteados tanto por parte del profesor como de algunos alumnos. En total, se registraron más de cincuenta interacciones en estas dos semanas bajo la referida etiqueta. Además, los alumnos que siguieron la actividad de la cuenta del profesor, acudieron a esta sesión con cierta ventaja, en tanto que este ya había tuiteado desde su cuenta una noticia referente al supuesto práctico planteado.

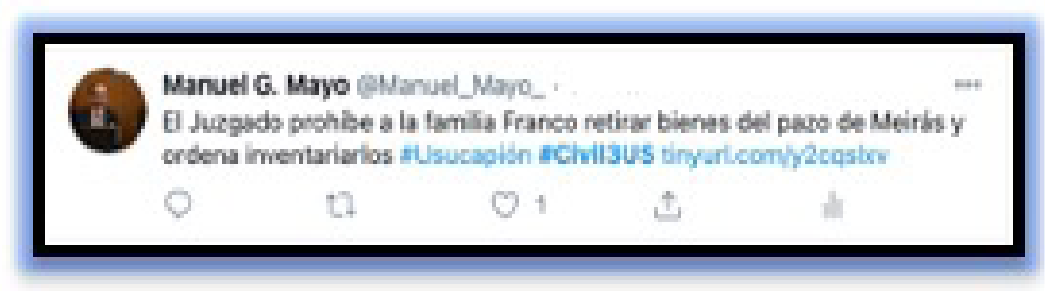

Figura 2. Tuit publicado por el profesor en relación con el supuesto práctico

Ciclos de Mejora en el Aula (2020). Experiencias de Innovación Docente de la US Esta obra se distribuye con la licencia Creative Commons 
Actividad 10: cuestionario final

Una vez desarrollada toda la materia prevista, vuelvo a pasar el mismo cuestionario que pasé el primer día de aplicación del CIMA, a fin de realizar una comparativa de los resultados.

\section{Actividad 11: test de autoevaluación}

Hasta ahora, hemos podido evaluar los conocimientos adquiridos por los alumnos en estas dos semanas. A efectos de cuantificar, procedo finalmente a realizar un cuestionario tipo test a través de la plataforma virtual. Se compone de diez preguntas, que a cada alumno le aparecen en distinto orden. Asimismo, también aparece en distinto orden las respuestas de cada pregunta y, una vez se pasa de pregunta, no se puede volver atrás. Les concedo doce minutos para la realización del test. A razón de 0,05 puntos por pregunta válida, quien las acierte todas puede llegar a obtener 0,5 de los 3 puntos totales que dedico a la evaluación continua en la calificación final de la asignatura. El resultado de este test realizado fue bastante más positivo que los otros test realizados hasta el momento.

\section{Evaluación del aprendizaje de los estudiantes}

Como se ha indicado anteriormente, a fin de evaluar el aprendizaje de los alumnos, llevo a cabo un análisis exhaustivo de los cuestionarios inicial y final cumplimentado por cada uno de ellos. He decir que, existiendo varios alumnos que solo habían realizado el cuestionario inicial o el final, y no ambos, tuve que limitarme a realizar este análisis comparativo atendiendo únicamente a los alumnos que habían estado presentes en ambas sesiones y que, por lo tanto, habían realizado el cuestionario en ambas ocasiones (16 alumnos), pues solo así podríamos llegar a realizar una evaluación concluyente a fin de tomar futuras decisiones.

Aunque el cuestionario inicial estaba compuesto por un total de diez preguntas, el análisis comparativo lo realizo

Ciclos de Mejora en el Aula (2020). Experiencias de Innovación Docente de la US Esta obra se distribuye con la licencia Creative Commons 
en base a la dos últimas preguntas, por dos razones, fundamentalmente: en primer lugar, porque de hacerlo con todas las preguntas sería un análisis excesivamente amplio; en segundo lugar, porque -como ya se ha indicadolas dos últimas preguntas -que conforman, a su vez, el quinto y último de los bloques del mapa de contenidoaglutinan a todas las preguntas anteriores, siendo, a su vez, las más amplias y las que más margen de reflexión otorgan.

En base a las respuestas de los alumnos en ambas preguntas y en ambos cuestionarios, realizo una escalera de aprendizaje para cada una de estas preguntas, con cuatro escalones en cada una de ellas correspondientes a los cuatro niveles en los que, en función de las respuestas, podrían dividirse los alumnos por sus conocimientos. Posteriormente, realizo un cuadro comparativo de la evolución de cada alumno a lo largo de estas dos semanas partiendo de sus respuestas en uno y otro cuestionario.

PREGUNTA 9: ¿Puede adquirirse la propiedad de una cosa mueble de alguien que no es el propietario? ¿Cuál cree que es el fundamento de que eso sea así?

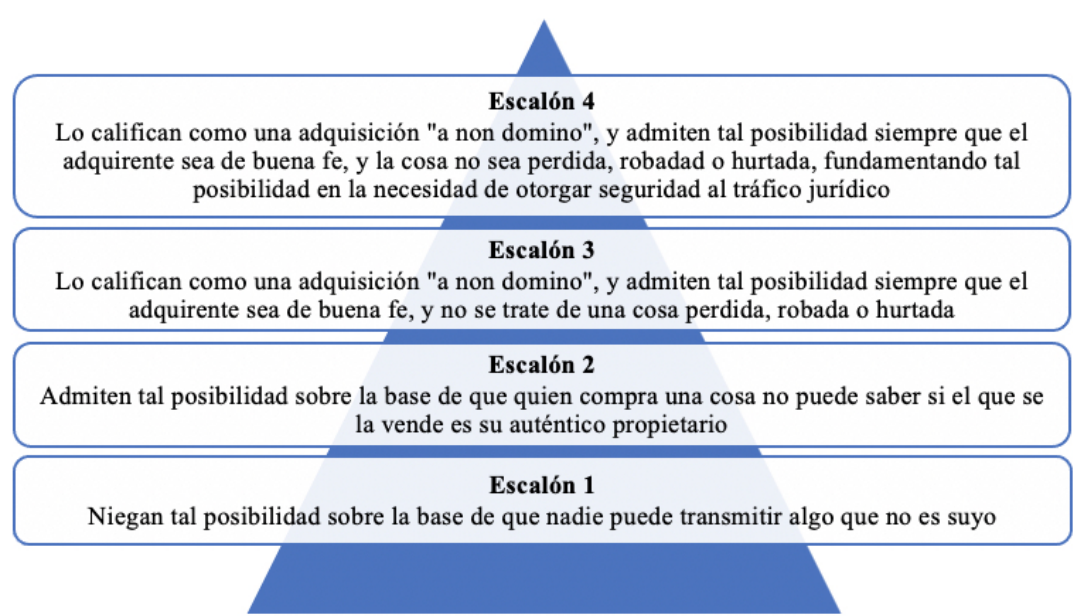

Figura 3. Escalera de aprendizaje en relación con la pregunta número 9

Ciclos de Mejora en el Aula (2020). Experiencias de Innovación Docente de la US Esta obra se distribuye con la licencia Creative Commons 
PREGUNTA 10: ¿Puede adquirirse la propiedad de una cosa a través de su mera posesión? ¿Cuál cree que es el fundamento de que eso sea así?

\section{Escalón 4}

Admiten tal posibilidad, reconocen que se denomina "usucapión" y reconocen la existencia tanto de una usucapión ordinaria como de una extraordinaria con distintos requisitos y plazos.

Justifican, asimismo, que esto sea así en el interés general y la seguridad del tráfico jurídico, en base a la eficacia de la apariencia

\section{Escalón 3}

Admiten tal posibilidad, reconocen que se denomina "usucapión" y reconocen la existencia tanto de una usucapión ordinaria como de una extraordinaria con distintos requisitos y plazos

Escalón 2

Admiten tal posibilidad siempre que el adquirente sea de buena fe

\section{Escalón 1}

Niegan tal posibilidad si no existe ningún negocio jurídico transmisivo de la propiedad; o admiten tal posibilidad solo si se trata de una "res nullius"

Figura 4. Escalera de aprendizaje en relación con la pregunta número 10

Tabla 3. Análisis de la evaluación de cada alumno en base a las respuestas de las preguntas núm. 9 y $10(\mathrm{Cl}$ : cuestionario inicial; CF: cuestionario final; E: escalón)

\begin{tabular}{|c|c|c|c|c|c|c|}
\cline { 2 - 7 } \multicolumn{1}{c|}{} & \multicolumn{3}{c|}{ PREGUNTA NÚM. 1 } & \multicolumn{3}{c|}{ PREGUNTA NÚM. 2 } \\
\cline { 2 - 7 } \multicolumn{1}{c|}{} & Cl & CF & PROGRESO & C1 & CF & PROGRESO \\
\hline Alumno 1 & E2 & E4 & +2 & E2 & E3 & +1 \\
\hline Alumno 2 & E1 & E2 & +1 & E1 & E3 & +2 \\
\hline Alumno 3 & E1 & E3 & +2 & E2 & E4 & +2 \\
\hline Alumno 4 & E1 & E3 & +2 & E2 & E3 & +1 \\
\hline Alumno 5 & E2 & E4 & +2 & E1 & E4 & +3 \\
\hline Alumno 6 & E2 & E4 & +2 & E2 & E3 & +1 \\
\hline Alumno 7 & E1 & E2 & +1 & E1 & E2 & +1 \\
\hline Alumno 8 & E1 & E3 & +2 & E3 & E3 & 0 \\
\hline Alumno 9 & E2 & E3 & +1 & E3 & E4 & +1 \\
\hline Alumno 10 & E2 & E3 & +1 & E1 & E4 & +3 \\
\hline Alumno 11 & E1 & E4 & +3 & E1 & E3 & +2 \\
\hline
\end{tabular}

Ciclos de Mejora en el Aula (2020). Experiencias de Innovación Docente de la US Esta obra se distribuye con la licencia Creative Commons 


\begin{tabular}{|l|c|c|c|c|c|c|}
\hline Alumno 12 & E1 & E3 & +2 & E1 & E2 & +1 \\
\hline Alumno 13 & E1 & E3 & +2 & E2 & E4 & +2 \\
\hline Alumno 14 & E2 & E2 & 0 & E1 & E4 & +3 \\
\hline Alumno 15 & E1 & E4 & +3 & E1 & E2 & +1 \\
\hline Alumno 16 & E1 & E3 & +2 & E1 & E3 & +2 \\
\hline
\end{tabular}

\begin{tabular}{|c|c|c|c|c|c|c|c|c|}
\cline { 2 - 9 } \multicolumn{1}{c|}{} & \multicolumn{3}{c|}{ CUESTIONARIO INICIAL (CI) } & \multicolumn{4}{c|}{ CUESTIONARIO FINAL (CF) } \\
\cline { 2 - 10 } \multicolumn{1}{c|}{} & E1 & E2 & E3 & E4 & E1 & E2 & E3 & E4 \\
\hline PREGUNTA 1 & 10 & 6 & 0 & 0 & 0 & 3 & 8 & 5 \\
\hline PREGUNTA 2 & 9 & 5 & 2 & 0 & 0 & 3 & 7 & 6 \\
\hline
\end{tabular}

Del análisis de los datos reflejados en la tabla comparativa anterior, podemos extraer conclusiones interesantes a fin de, a corto plazo, saber en qué partes de la explicación teórica pueden haber quedado lagunas y, a largo plazo, saber si alguna parte del temario explicado hemos de enfocarla de otra manera.

Como puede observarse, en la pregunta número 9 -referente a la adquisición a non domino- nadie partía de un escalón que no fuese el primer o el segundo, lo cual podría ser motivo de que es una materia que les es bastante ajena a su formación inicial antes de abordar su explicación; en la pregunta número 10 -referente a la usucapión- hay algunos alumnos que parten desde un inicio del escalón tercero, lo cual podría ser síntoma de que se trata de una materia de la que tienen conocimiento por su formación en cursos anteriores o bien, incluso, en los que su aplicación de la lógica en el cuestionario inicial se ha correspondido en mayor medida con la respuesta otorgada desde el Derecho.

En cuanto al cuestionario final, puede advertirse cómo el nivel de conocimiento de los alumnos, en cómputo global, es bastante similar en ambas preguntas. Solo tres alumnos se encuentran aún en el segundo escalón en cada una de las preguntas analizadas, mientras que el resto se sitúan en el escalón tercero o cuarto $-y$ último-. Ello es 
indicativo de que no ha habido una parte de la materia en la que el profesor no haya conseguido llegar al alumno de una forma tan eficaz como en el resto de la materia.

\section{Evaluación del CIMA}

Una vez concluida la última sesión, dialogué con los estudiantes en el aula -virtual y presencial- a fin de que me transmitieran sus impresiones al respecto. Si bien reconocieron que, en un principio, les costó habituarse a la nueva dinámica, y hacerse partícipes de la clase atendiendo a la dinámica imperante hasta entonces, reconocen que el sistema aplicado durante estas dos semanas les ha servido especialmente para involucrarse en la explicación, sentirse partícipes de la misma y valorar el sentido práctico de todo cuanto escuchaban.

A nivel personal, intentaré aplicar esta metodología de una forma más continua, de forma tal que, haciéndolo de forma paulatina, consiga desarrollar toda la materia aplicando este diseño de clase. He de decir que me ha parecido especialmente atrayente la idea de utilizar música o vídeo para captar la atención del alumno al comienzo de la clase, pues fue especialmente gratificante la utilización de una marcha de Semana Santa al comienzo de unas clases, lo cual me ha demostrado, posteriormente, que los alumnos no se hayan olvidado de cuanto se explicó en el transcurso de esa sesión.

No obstante, hay aspectos que he de tener en cuenta a fin de diseñar futuros CIMA en mi asignatura. Así, en primer lugar, he de aliviar - aún más de contenido- mis sesiones, o hacer ciclos más breves, pues el mapa de contenido diseñado y aplicado ha resultado, quizás, demasiado extenso, hasta el punto de que, si bien ha habido tiempo para hacer todo cuanto estaba programado, se ha hecho, en ocasiones, de una forma especialmente veloz a fin de dar cabida a toda la materia. He de realizar un cribado aún más minucioso de la materia a ver en clase para que las

Ciclos de Mejora en el Aula (2020). Experiencias de Innovación Docente de la US Esta obra se distribuye con la licencia Creative Commons 
sesiones resulten menos densas. Además, he de replantear especialmente las sesiones de dos horas para que, en todo caso, haya variedad de actividades y no ocurra como me ocurrió en la sesión número 3, que fue eminentemente teórica y pudo resultar más árida que las demás. Asimismo, considero que el cuestionario inicial-final a realizar ha de se aún más breve, pues, en la práctica me he percatado de que, incluso, con las dos últimas preguntas habría sido suficiente para ser consciente del grado de conocimiento del alumno sobre la materia.

Ciclos de Mejora en el Aula (2020). Experiencias de Innovación Docente de la US Esta obra se distribuye con la licencia Creative Commons 
Palabras claves: Derechos reales e hipotecario, grado en Derecho, docencia universitaria, experimentación docente universitaria, problemas prácticos.

Key words: Real rights and mortgage law, law degree, university teaching, university teaching experimentation, practical problems

\section{Referencias bibliográficas}

Bain, K. (2007). Lo que hacen los mejores profesores universitarios. Valencia: Publicaciones Universidad de Valencia.

Finkel, D. (2008). Dar clase con la boca cerrada. Valencia: Publicaciones Universidad de Valencia.

Porlán, R. (Coord.) (2017). Enseñanza Universitaria. Cómo mejorarla. Madrid: Ediciones Morata.

Ciclos de Mejora en el Aula (2020). Experiencias de Innovación Docente de la US Esta obra se distribuye con la licencia Creative Commons 